\title{
SOME WOLSTENHOLME TYPE CONGRUENCES
}

\author{
ROMEO MEŠTROVIĆ
}

Abstract. In this paper we give an extension and another proof of the following Wolstenholme's type curious congruence established in 2008 by J. Zhao. Let $s$ and $l$ be two positive integers and let $p$ be a prime such that $p \geq l s+3$. Then

$$
H\left(\{s\}^{l} ; p-1\right) \equiv S\left(\{s\}^{l} ; p-1\right) \equiv\left\{\begin{array}{lll}
-\frac{s(l s+1) p^{2}}{2(l s+2)} B_{p-l s-2} & \left(\bmod p^{3}\right) & \text { if } 2 \nmid l s \\
(-1)^{l-1} \frac{s p}{l s+1} B_{p-l s-1} & \left(\bmod p^{2}\right) & \text { if } 2 \mid l s .
\end{array}\right.
$$

As an application, for given prime $p \geq 5$, we obtain explicit formulae for the sum $\sum_{1 \leq k_{1}<\cdots<k_{l} \leq p-1} 1 /\left(k_{1} \cdots k_{l}\right)\left(\bmod p^{3}\right)$ if $k \in\{1,3, \ldots, p-2\}$, and for the sum $\sum_{1 \leq k_{1}<\cdots<k_{l} \leq p-1} 1 /\left(k_{1} \cdots k_{l}\right)\left(\bmod p^{2}\right)$ if $k \in\{2,4, \ldots, p-3\}$.

\section{IntRoduction AND BASIC RESUlts}

Our investigations are motivated by some recent results to multiple harmonic sums obtained by J. Zhao [12], Zhou and Cai [13]. These results are in fact, variations and generalizations of Wolstenholme's theorem. For more information on extensions and generalizations of Wolstenholme's theorem, see [5], [9], [10] and [11].

Throughout this paper we use the following definitions and notations.

For $n \in \mathbb{N}, l \in \mathbb{N}$ and $\mathbf{s}:=\left(s_{1}, \ldots, s_{l}\right) \in \mathbb{N}^{l}$, define the finite harmonic sum

$$
H(\mathbf{s} ; n):=H\left(s_{1}, \ldots, s_{l} ; n\right)=\sum_{1 \leq k_{1}<\cdots<k_{l} \leq n} \frac{1}{k_{1}^{s_{1}} \cdots k_{l}^{s_{l}}} .
$$

By convention we set $H(\mathbf{s} ; r)=0$ for $r=0, \ldots, l-1$. Further, we define the sum

$$
S(\mathbf{s} ; n):=S\left(s_{1}, \ldots, s_{l} ; n\right)=\sum_{1 \leq k_{1} \leq \cdots \leq k_{l} \leq n} \frac{1}{k_{1}^{s_{1}} \cdots k_{l}^{s_{l}}} .
$$

If $s_{1}=\ldots=s_{l}=s$ then $H(\mathbf{s} ; n)$ is a homogeneous harmonic sum. In this case, we shall denote such a sum by $H\left(\{s\}^{l} ; n\right)$, and hence

$$
H\left(\{s\}^{l} ; n\right)=\sum_{1 \leq k_{1}<\cdots<k_{l} \leq n} \frac{1}{\left(k_{1} \cdots k_{l}\right)^{s}} .
$$

$M S C$ (2010): primary 11B65; secondary 11A07, 05A10.

Keywords: multiple harmonic sum, Wolstenholme's theorem, Bernoulli numbers, Stirling numbers. 
In particular, we write $H(s ; n)$ instead of $H\left(\{s\}^{1} ; n\right)$, that is,

$$
H(s ; n)=\sum_{1 \leq k \leq n} \frac{1}{k^{s}} .
$$

Analogously, we define $S\left(\{s\}^{l} ; n\right)$ and $S(s ; n)$ related to the sums $S(\mathbf{s} ; n)$.

Recall that Stirling numbers $S t(n, j)$ of the first kind are defined by the expansion

$$
x(x+1)(x+2) \cdots(x+n-1)=\sum_{j=1}^{n} S t(n, j) x^{j} .
$$

It is easy to see that for all $j=1, \ldots, n-1$,

$$
\operatorname{St}(n, j)=\sum_{1 \leq k_{1}<\cdots<k_{n-j} \leq n-1} k_{1} \cdots k_{n-j},
$$

and that

$$
S t(n, j+1)=(n-1) ! \cdot H\left(\{1\}^{j} ; n-1\right) .
$$

For example, $\operatorname{St}(n, n)=1, S t(n, n-1)=n(n-1) / 2$, and $S t(n, 1)=(n-1) !$.

Further, for any nonnegative integers $j$ and $n \geq 1, j$ th power-sum symmetric function is defined as

$$
P(n, j)=\sum_{k=1}^{n} k^{j} .
$$

By convention we set $P(0, j)=0$ for all $j \geq 0$.

By Wolstenholme's theorem (see, e.g., [6, p. 89]), if $p$ is a prime greater than 3 , then the numerator of the fraction

$$
H(1, p-1)=1+\frac{1}{2}+\frac{1}{3}+\cdots+\frac{1}{p-1}
$$

written in reduced form, is divisible by $p^{2}$.

Denote by $\mathfrak{p}$ the parity of $m$ which is 1 if $m$ is odd and 2 if $m$ is even. Bayat proved

Theorem A ([1, Theorem 3]; also see Remark 2.3 in [12]). For any positive integer $s$ and a prime $p \geq s+3$ we have

$$
H(s ; p-1) \equiv 0 \quad\left(\bmod p^{\mathfrak{p}(s+1)}\right) .
$$

Zhao in $[12$, p. 74$]$ reported that one can find on the Internet the following generalization of Wolstenholme's theorem by Bruck [2], although no proof is given here.

Theorem B ([12, Theorem 1.2]). For any prime number $p \geq 5$ and positive integers $l=1, \ldots, p-3$, we have

$$
S t(p, l+1) \equiv 0 \quad\left(\bmod p^{\mathfrak{p}(l+1)}\right)
$$

and

$$
H\left(\{1\}^{l} ; p-1\right) \equiv 0 \quad\left(\bmod p^{\mathfrak{p}(l+1)}\right)
$$


The Bernoulli numbers $B_{k}$ are defined by the generating function

$$
\sum_{k=0}^{\infty} B_{k} \frac{x^{k}}{k !}=\frac{x}{e^{x}-1} .
$$

It is easy to find the values $B_{0}=1, B_{1}=-\frac{1}{2}, B_{2}=\frac{1}{6}, B_{4}=-\frac{1}{30}$, and $B_{n}=0$ for odd $n \geq 3$. Furthermore, $(-1)^{n-1} B_{2 n}>0$ for all $n \geq 1$. These and many other properties can be found, for instance, in [7] and [3].

Recently, Zhao in [12] proved the following generalization of Theorem B to homogeneous multiple harmonic sums.

Theorem C ([12, Theorem 2.14]; cf. [12, Theorem 1.6]). Let $s$ and $l$ be two positive integers. Let $p$ be a prime such that $p \geq l s+3$. Then

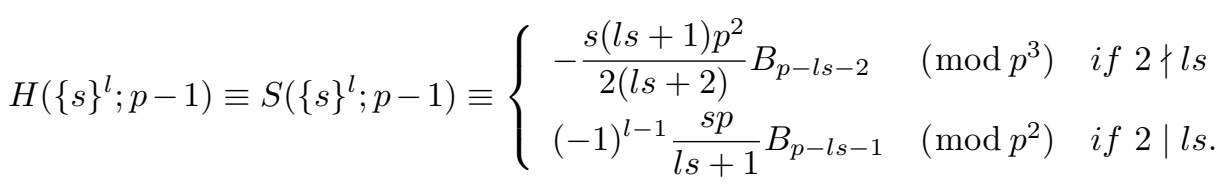

As an application, Zhao obtained the following result.

Theorem D ([12, Proposition 2.15]; also cf. [12, Theorem 1.5]). Let $s$ and $l$ be two positive integers. Let $p$ be a prime such that $p \geq l+2$ and $p-1$ divides none of $k s$ and $k s+1$ for $k=1, \ldots, l$. Then

$$
H\left(\{s\}^{l} ; p-1\right) \equiv S\left(\{s\}^{l} ; p-1\right) \equiv 0 \quad\left(\bmod p^{\mathfrak{p}(l s-1)}\right) .
$$

In particular, if $p \geq l s+3$, then the above is always true, and so $p \mid H\left(\{s\}^{l} ; p-1\right)$.

As noticed in [12, p. 85, Proof of Theorem 2.14], the above congruence for $H\left(\{s\}^{l} ; p-1\right)$ follows immediately from [13, Lemma 2$]$, while the above congruence for $S\left(\{s\}^{l} ; p-1\right)$ then follows from the equality (2.11) in [12] and induction on $l$.

In Section 2 we give another proof of the above congruence for $H\left(\{s\}^{l} ; p-1\right)$. Our result is as follows.

Theorem 1.1. Let $s$ and $l$ be two positive integers. Let $p$ be a prime such that $p \geq l s+3$. Then

$$
H\left(\{s\}^{l} ; p-1\right) \equiv(-1)^{l-1} \frac{H(l s ; p-1)}{l} \equiv\left\{\begin{array}{l}
-\frac{s(l s+1) p^{2}}{2(l s+2)} B_{p-l s-2}\left(\bmod p^{3}\right) \text { if } 2 \nmid l s \\
(-1)^{l-1} \frac{s p}{l s+1} B_{p-l s-1}\left(\bmod p^{2}\right) \text { if } 2 \mid l s .
\end{array}\right.
$$

Taking $s=1$ in Theorem 1.1, we obtain the following result.

Corollary 1.2. Let $p$ be a prime greater than 3 , and let $l$ be a positive integer such that $l \leq p-3$. Then

$$
H\left(\{1\}^{l} ; p-1\right):=\sum_{1 \leq k_{1}<\cdots<k_{l} \leq p-1} \frac{1}{k_{1} \cdots k_{l}} \equiv \begin{cases}-\frac{(l+1) p^{2}}{2(l+2)} B_{p-l-2} & \left(\bmod p^{3}\right) \text { if } 2 \nmid l \\ -\frac{p}{l+1} B_{p-l-1} & \left(\bmod p^{2}\right) \text { if } 2 \mid l .\end{cases}
$$


Corollary 1.3. Let $p$ be a prime greater than 3 , and let $l$ be a positive integer such that $2 \leq l \leq p-2$. Then

$$
S t(p, l) \equiv \begin{cases}\frac{l p^{2}}{2(l+1)} B_{p-1-l} & \left(\bmod p^{3}\right) \text { if } 2 \mid l \\ \frac{p}{l} B_{p-l} & \left(\bmod p^{2}\right) \text { if } 2 \nmid l .\end{cases}
$$

Proof. Since by Wilson theorem, $(p-1) ! \equiv-1(\bmod p)$, we get for all $l=$ $2, \ldots, p-2$

$$
S t(p, l)=(p-1) ! \cdot H\left(\{1\}^{l-1} ; p-1\right) \equiv-H\left(\{1\}^{l-1} ; p-1\right) \quad(\bmod p) .
$$

Therefore, both congruences follow immediately from the congruences given in Corollary 1.2.

Remark 1.4. Observe that Corollary 1.2 does not contain a related congruence for the Stirling number $S t(p, 1)=(p-1)$ !. In 1900 Glaisher [4] showed that $(p-1) ! \equiv p B_{p-1}-p\left(\bmod p^{2}\right)$.

In order to prove Theorem 1.1, we use Theorem A in the proof of Lemma 2.2 given in Section 2. Note that Theorem A is an immediate consequence of a classical result of E. Lehmer [8] given by Lemma 2.7. On the other hand, Theorem B is an immediate consequence of Corollaries 1.2 and 1.3 and the fact that by Lemma 2.5 , the denominator of the Bernoulli number $B_{l}$, written in reduced form, is not divisible by $p$ for each integer $l$ such that $0 \leq l \leq p-3$. Further, the congruence for $H\left(\{s\}^{l} ; p-1\right)$ in Theorem $\mathrm{C}$ is given by Theorem 1.1. Finally, note that (see Remark 2.3) the proof of Theorem D is the same as that of Lemma 2.2.

\section{Proof of Theorem 1.1}

For the proof of Theorem 1.1, we will need some auxiliary results.

Lemma 2.1. Let $n, s$ and $l$ be positive integers such that $l \geq 2$ and $n \geq l s$. Then

$$
\sum_{j=1}^{l-1}(-1)^{j-1} H(j s ; n) \cdot H\left(\{s\}^{l-j} ; n\right)=l H\left(\{s\}^{l} ; n\right)+(-1)^{l} H(l s ; n) .
$$

Proof. First note that (2.1) is trivially satisfied for $l=2$. For simplicity, here we write $H\left(\{\mathbf{s}\}^{l}\right)$ instead of $H\left(\{\mathbf{s}\}^{l} ; n\right)$, and denote

$$
\sigma(j)=\sum_{i=0}^{l-j} H(\underbrace{s, \ldots, s}_{i}, j s, \underbrace{s, \ldots, s}_{l-j} ; n), \quad j=1, \ldots l,
$$


whence we see that $\sigma(l)=H(l s ; n)$. Now if $l \geq 3$, then for all $j$ with $2 \leq j \leq l-1$, we have

$$
\begin{aligned}
H(j s) \cdot H\left(\{s\}^{l-j}\right) & =\left(\sum_{1 \leq k \leq n} \frac{1}{k^{j s}}\right)\left(\sum_{1 \leq k_{1}<\cdots<k_{l-j} \leq n} \frac{1}{\left(k_{1} \cdots k_{l-j}\right)^{s}}\right) \\
& =\sum_{k \notin\left\{k_{1}, \ldots, k_{l-j}\right\}}+\sum_{k \in\left\{k_{1}, \ldots, k_{l-j}\right\}} \\
& =\sum_{i=0}^{l-j} H(\underbrace{s, \ldots, s}_{i}, j s, \underbrace{s, \ldots, s}_{l-j-i}) \\
& +\sum_{i=0}^{l-j-1} H(\underbrace{s, \ldots, s}_{i},(j+1) s, \underbrace{s, \ldots, s}_{l-j-1-i}) \\
& =\sigma(j)+\sigma(j+1) .
\end{aligned}
$$

Furthermore, for $j=1$, we have

$$
\begin{aligned}
H(s) \cdot H\left(\{s\}^{l-1}\right) & =\left(\sum_{1 \leq k \leq n} \frac{1}{k^{s}}\right)\left(\sum_{1 \leq k_{1}<\cdots<k_{l-1} \leq n} \frac{1}{\left(k_{1} \cdots k_{l-1}\right)^{s}}\right) \\
& =\sum_{k \notin\left\{k_{1}, \ldots, k_{l-1}\right\}}+\sum_{k \in\left\{k_{1}, \ldots, k_{l-1}\right\}} \\
& =\sum_{i=0}^{l-1} H(\underbrace{s, \ldots, s}_{i}, s, \underbrace{s, \ldots, s}_{l-1-i})+\sum_{i=0}^{l-1} H(\underbrace{s, \ldots, s}_{i}, 2 s, \underbrace{s, \ldots, s}_{l-2-i}) \\
& =l H\left(\{s\}^{l}\right)+\sigma(2) .
\end{aligned}
$$

The above two equalities imply

$$
\begin{aligned}
\sum_{j=1}^{l-1}(-1)^{j-1} H(j s) \cdot H\left(\{s\}^{l-j}\right) & =l H\left(\{s\}^{l}\right)+\sigma(2)+\sum_{j=2}^{l-1}(-1)^{j-1}(\sigma(j)+\sigma(j+1)) \\
& =l H\left(\{s\}^{l}\right)+\sigma(2)+\sum_{j=2}^{l-1}(-1)^{j-1}(\sigma(j)+\sigma(j+1)) \\
& =l H\left(\{s\}^{l}\right)+\sigma(2)-\sigma(2)+(-1)^{l-1} \sigma(l) \\
& =l H\left(\{s\}^{l}\right)+(-1)^{l} H(l s ; n),
\end{aligned}
$$

as desired.

The following lemma is an extension of the congruence for harmonic sums $H\left(\{1\}^{l} ; p-1\right)$ given by Theorem B. This is in fact the congruence for $H\left(\{s\}^{l} ; p-1\right)$ from Theorem $\mathrm{D}$ when $p \geq l s+3$.

Lemma 2.2 (cf. Theorem C). Let $s$ and $l$ be two positive integers, and let $p$ be a prime such that $p \geq l s+3$. Then $p^{2} \mid H\left(\{s\}^{l} ; p-1\right)$ if $l s$ is odd, and $p \mid H\left(\{s\}^{l} ; p-1\right)$ if $l s$ is even. 
Proof. Putting $n=p-1$ in (2.1) of Lemma 2.1, we obtain

$$
\begin{aligned}
& \sum_{j=1}^{l-1}(-1)^{j-1} H(j s ; p-1) \cdot H\left(\{s\}^{l-j} ; p-1\right) \\
& \quad+(-1)^{l-1} H(l s ; p-1)=l H\left(\{s\}^{l} ; p-1\right) .
\end{aligned}
$$

We proceed by induction on the sum $\sigma:=l+s \geq 2$. If $\sigma=2$ then $l=s=1$, and $p^{2} \mid H(1 ; p-1)$ by Wolstenholme's theorem. Now suppose that the assertion is true for some $\sigma$ with $\sigma \geq 2$. This means that $p^{2} \mid H\left(\left\{s^{\prime}\right\}^{l^{\prime}} ; p-1\right)$ whenever $l^{\prime}$ and $s^{\prime}$ are both odd such that $l^{\prime}+s^{\prime} \leq \sigma$ and $p \geq l^{\prime} s^{\prime}+3$, and that $p \mid H\left(\left\{s^{\prime}\right\}^{l^{\prime}} ; p-1\right)$ whenever $l^{\prime} s^{\prime}$ is even such that $l^{\prime}+s^{\prime} \leq \sigma$ and $p \geq l^{\prime} s^{\prime}+3$ In order to prove the assertion for all pairs $l$ and $s$ with $l+s=\sigma$ and $p \geq l s+3$, we consider the following two cases.

Case 1. $l s$ is odd; that is both integers $l$ and $s$ are odd. Then, for odd $j$ with $1 \leq j \leq l-1$, we have $s+(l-j)=\sigma-j<\sigma$ and $s(l-j)$ is even. Therefore, by the inductive hypothesis, $p \mid H\left(\{s\}^{l-j}\right.$. Furthermore, for such a $j$, by Theorem A, $p^{2} \mid H(j s ; p-1)$.

Similarly, if $j$ is even with $1 \leq j \leq l-1$, we also have $s+(l-j)=\sigma-j<\sigma$, and $s(l-j)$ is odd. Thus, by the inductive hypothesis, $p^{2} \mid H\left(\{s\}^{l-j}\right.$. Furthermore, for such a $j$, by Theorem A, $p \mid H(j s ; p-1)$.

Hence, in both cases it follows that $p^{3} \mid H(j s ; p-1) \cdot H\left(\{s\}^{l-j} ; p-1\right)$. This together with the fact that, by Theorem A, $p^{3} \mid H(l s ; p-1)$, implies that the sum on the right hand side of $(2.2)$ is divisible by $p^{3}$. Therefore, $p^{3} \mid l H\left(\{s\}^{l} ; p-1\right)$, whence, because $l \leq l s \leq p-3$, it follows that $p^{3} \mid H\left(\{s\}^{l} ; p-1\right)$. This concludes the inductive proof when $l s$ is odd.

Case 2. $l s$ is even. Then in the same way as in the first case, we obtain by induction on the sum $l+s$ that $p^{2} \mid H\left(\{s\}^{l} ; p-1\right)$.

This completes the inductive proof.

Remark 2.3. Observe that the above proof holds if we replace the condition $p \geq l s+3$ of the Lemma by the following conditions of Theorem D: $p \geq l+2$ and $p-1$ divides none of $k s$ and $k s+1$ for $k=1, \ldots, l$. In other words, Theorem D can be proved in the same manner as Lemma 2.2 .

We are now ready to state the following

Proposition 2.4. Let $s$ and $l$ be two positive integers, and let $t=\max \{1, l-1\}$. Let $p$ be a prime such that $p \geq t s+3$. Then

$$
l H\left(\{s\}^{l} ; p-1\right) \equiv(-1)^{l-1} H(l s ; p-1) \begin{cases}\left(\bmod p^{3}\right) & \text { if } 2 \nmid l s \\ \left(\bmod p^{2}\right) & \text { if } 2 \mid l s .\end{cases}
$$

Proof. If $l=1$ and $p \geq s+3$, then the above congruences reduce to the congruence for $H(s ; p-1)$ given in Theorem A. If $l \geq 2$ and $p \geq(l-1) s+3$, by (2.2) of the proof of Lemma 2.2, we have

$$
\sum_{j=1}^{l-1}(-1)^{j-1} H(j s ; p-1) \cdot H\left(\{s\}^{l-j} ; p-1\right)=l H\left(\{s\}^{l} ; p-1\right)-(-1)^{l} H(l s ; p-1) .
$$


From the proof of Lemma 2.2, we see that each term of the sum on the left hand side of the above identity is divisible by $p^{3}$ if $l s$ is odd, and by by $p^{2}$ if $l s$ is even. Clearly, this fact implies both congruences from our Proposition.

Lemma 2.5. Let $p \geq 3$ be a prime, and let $s$ be any even integer such that $0 \leq s \leq p-3$. Then the denominator of the Bernoulli number $B_{s}$, written in reduced form, is not divisible by $p$.

Proof. If $p=3$, then $s=0$, that is, $B_{0}=1$. Suppose now that $p \geq 5$. It is well known (see [7]) that Bernoulli numbers can be defined recursively as

$$
B_{s}=-\frac{1}{s+1} \sum_{i=0}^{s-1}\left(\begin{array}{c}
s+1 \\
i
\end{array}\right) B_{i}
$$

Now, by induction on even $s$ with $0 \leq s \leq p-3$, the above equality immediately implies that the denominator of $B_{s}$, written in reduced form, is not divisible by $p$.

Remark 2.6. The above lemma is an immediate consequence of the von Staudt-Clausen theorem, which asserts that $B_{2 m}+\sum_{p-1 \mid 2 m} 1 / p$ is an integer for all $m \in \mathbb{N}$, where the summation is over all primes $p$ such that $p-1 \mid 2 m$ (see, for example, [7, p. 233, Theorem 3]). If $B_{2 m}=N_{2 m} / D_{2 m}$ with $\operatorname{gcd}\left(N_{2 m}, D_{2 m}\right)=1$, then, by this result, it follows that the denominator $D_{2 m}$ of $B_{2 m}$ is given by

$$
D_{2 m}=\prod_{\substack{p \text { prime } \\ p-1 \mid 2 m}} p
$$

whence Lemma 2.5 follows.

The following result is closely related to congruences of Glaisher [4], as quoted and proved by E. Lehmer (two congruences after (16) in [8]).

Lemma 2.7 ([8]; also cf. [12, Theorem 2.8]). Let $p \geq 5$ be a prime, and let $m$ be any integer such that $1 \leq m \leq(p-3) / 2$. Then

$$
H(2 m-1 ; p-1):=\sum_{k=1}^{p-1} \frac{1}{k^{2 m-1}} \equiv \frac{m(1-2 m) p^{2}}{2 m+1} B_{p-1-2 m} \quad\left(\bmod p^{3}\right)
$$

and

$$
H(2 m ; p-1):=\sum_{k=1}^{p-1} \frac{1}{k^{2 m}} \equiv \frac{2 m p}{2 m+1} B_{p-1-2 m} \quad\left(\bmod p^{2}\right) .
$$

Proof. Consider the case when $l s$ is odd, that is, when both $l$ and $s$ are odd. By the first congruence of Proposition 2.4 and the congruence (2.3) of the above lemma with $l s=2 m-1$, we obtain

$$
\begin{aligned}
& H\left(\{s\}^{l} ; p-1\right) \equiv \frac{H(l s ; p-1)}{l} \quad\left(\bmod p^{3}\right) \\
& \equiv-\frac{1}{l} \frac{s l(l s+1) p^{2}}{2(l s+2)} B_{p-l s-2} \quad\left(\bmod p^{3}\right) \\
& =-\frac{s(l s+1) p^{2}}{2(l s+2)} B_{p-l s-2} \quad\left(\bmod p^{3}\right) \text {. }
\end{aligned}
$$


Similarly, using the second congruence of Proposition 2.4 and the congruence (2.4) of the above lemma with $l s=2 m$, we obtain the congruence of Theorem 1.1 for even $l s$.

\section{REFERENCES}

[1] M. Bayat, A generalization of Wolstenholme's Theorem, Amer. Math. Monthly 104 (1997), $557-560$.

[2] R. Bruck, Wolstenholme's theorem, Stirling numbers, and binomial coefficients, available at mathlab.usc.edu/ bruck/research/stirling/.

[3] K. Dilcher, L. Skula and I.Sh. Slavutsky: Bernoulli Numbers Bibliography (17131990), Queen's papers in Pure and Appl. Math., vol. 87, 175 pp.; Appendix, Queen's University, Kingston, Ontario, 1991, updated on-line version: http://www.mathstat.dal.ca/ dilcher/bernoulli.html. Zbl 0741.11001.

[4] J.W. L. Glaisher, On the residues of the sums of products of the first $p-1$ numbers and their powers, to modulus $p^{2}$ or $p^{3}$, Quart. J. Math. 31 (1900), 321-353.

[5] A. Granville, Arithmetic properties of binomial coefficients. I. Binomial coefficients modulo prime powers, in Organic mathematics (Burnaby, BC, 1995), CMS Conf. Proc., 20, Amer. Math. Soc., Providence, RI, 1997, 253-276.

[6] G. H. Hardy and E. M. Wright, An Introduction to the Theory of Numbers, Clarendon press, Oxford, 1980.

[7] K. Ireland and M. Rosen, A Classical Introduction to Modern Number Theory, SpringerVerlag, New York, 1982.

[8] E. Lehmer, On congruences involving Bernoulli numbers and the quotients of Fermat and Wilson, Ann. Math. 39 (1938), 350-360.

[9] R. Meštrović, A note on the congruence $\left(\begin{array}{c}n p^{k} \\ m p^{k}\end{array}\right) \equiv\left(\begin{array}{c}n \\ m\end{array}\right)\left(\bmod p^{r}\right)$, Czechoslovak Math. J. 62 (2012), 59-65.

[10] R. Meštrović, On the mod $p^{7}$ determination of $\left(\begin{array}{c}2 p-1 \\ p-1\end{array}\right)$, accepted for publication in Rocky Mountain J. Math., preprint arXiv:1108.1174 [mathNT], 2011.

[11] R. Meštrović, Wolstenholme's theorem: Its Generalizations and Extensions in the last hundred and fifty years (1862-2012) preprint arXiv: 0911.4433v3 [math.NT], 2011.

[12] J. Zhao, Wolstenholme type theorem for multiple harmonic sum, Int. J. Number Theory 4 (2008), 73-106.

[13] X. Zhou and T. Cai, A generalization of a curious congruence on harmonic sums, Proc. Amer. Math. Soc. 135 (2007), 1329-1333.

Romeo Meštrović, Maritime Faculty, University of Montenegro, Dobrota 36, 85330 Kotor, Montenegro

e-mail: romeo@ac.me 